\section{G310(P) AUDIT OF DISCHARGE LETTER DIAGNOSIS AND ITS EFFECT ON CLINICAL CODING}

S Liew, S Bandi. Paediatrics, Leicester Royal Infirmary, Leicester, UK

10.1136/archdischild-2015-308599.287

Introduction Discharge diagnoses can have significant implications on patient management from a clinical governance point of view. It can further impact the clinical coding with a risk of potential financial loss to the service provider. Accurate clinical coding also contributes to appropriate data collection and analysis in audit and research.

Aims To look at the accuracy of discharge letter primary diagnosis and co-morbidities

To look at the relation between clinical coding and potential financial loss

Methods We did a retrospective review of 50 discharge letters randomly selected from the paediatric wards in August 2014.

An excel spread sheet was used to collect the data which included: date of admission and discharge, discharge diagnosis and any co-morbidity. The accuracy of the diagnosis was ascertained by looking through the patient notes and cross-checking them with clinical coders and against the ICD-10 manual.

Results 13 out of 50 discharge letters (26\%) had inaccurate diagnosis.

6 out of 50 case notes (12\%) had co-morbidities that were not included on discharge letters.

We looked thorough seven case notes' discharge diagnosis (7/13) in detail and compared the financial tariff between the accurate diagnosis and the one written on the discharge letter. We estimated an income loss of $£ 1,359$.

Discussion Coding accuracy on average is high in the United Kingdom, especially for operations and procedures. ${ }^{1}$

Inaccurate coding can have significant financial implications. ${ }^{2}$ There is a need to raise the awareness of the importance of accurate clinical coding for all clinicians.

Conclusion

1. Our audit demonstrated that inaccurate diagnosis can have significant financial implications

2. A Do's and Don'ts table was designed and circulated to all the clinical staff (see Figure 1)

3. We aim to raise the awareness of clinical coding during junior doctors induction and aim to re-audit in 6 months' time.

\section{Discharge letter - Clinical Coding}

wopD to UsE
- Diagnosis/ working
diagnosis
$\checkmark$ Clinically relevant
- Probable
- Treated As
- Presumed

DO NOT USE

* ?

* Differentials

* Likely/Most likely

* Possible

* Investigated As

* Impression

* Maybel Felt to be

* Suspected

\section{REFERENCES}

1 Campbell SE, Campbell MK, Grimshaw JM, Walker AE. A systematic review of discharge coding accuracy. J Public Health Med. 2001; 23(3):205-11

2 Stevens S, Unwin CE, Codde JP. A review of hospital medical record audits: implications for funding and training. Aust Health Rev. 1998; 21(3):78-91

\section{G311(P) AUDITOF SPINAL ULTRASOUND (SU) FOR NEONATAL SACRAL DIMPLES}

'D Pandya, ${ }^{2} \mathrm{O}$ Kayode, ${ }^{3} \mathrm{~A}$ Gandhi. ${ }^{1}$ Gastroenterology, The Dudley Group of Hospitals, NHS Foundation Trust, Birmingham, UK; ${ }^{2}$ General Practice, Barts Health NHS Trust, London, UK; ${ }^{3}$ Paediatrics, Heart of England Foundation Trust, Birmingham, UK

\subsection{6/archdischild-2015-308599.288}

Background Isolated sacral dimples are common and it is increasingly recognised that most are not linked to spinal dysraphism. Based on their features they can be classified into low risk and high risk. The Royal college of Radiologists guidance states that the high risk dimples (large, base not visible, $>25 \mathrm{~mm}$ from anus or $>5 \mathrm{~mm}$ from midline or associated with additional stigmata of spinal dysraphism) should be investigated with SU.

Objective To determine whether hospital guidelines specific to sacral dimples are being followed and to ascertain the yield of SU. Method A cohort of neonates from the entire Trust who received a spinal ultrasound scan due to the presence of a sacral dimple between 2007-2011 were included. Information was gathered from clinical letters, referral forms, and the presence or absence of a spinal abnormality was assessed by evaluating the ultrasound scan reports.

Results 94 neonates underwent SU for sacral dimple.

89 (95\%) infants with sacral dimple underwent SU, 4 (4\%) SUS with additional MRI scanning and 1 (1\%) SUS with plain radiography.

$63(66 \%)$ of sacral dimples were described in the referral as high risk, $7(7 \%)$ as low risk and $24(25 \%)$ sacral dimples had too little detail/description in their referrals to determine the risk.

Therefore, over the 3 year duration, only $7 \%$ of sacral dimples were low risk in nature so were ultra-sounded against hospital guidelines.

'Indeterminate dimples' had limited description on the radiology request form, assuming these were also low risk in nature, $31(33 \%)$ sacral dimples were ultra-sounded against guidelines.

$13 \%$ of high risk sacral dimples in this audit revealed a spinal abnormality upon imaging. No abnormality was detected in cases not classed as high risk. This is in line with other reports and supports the current practice of selective SU in newborns with high risk dimples.

Conclusion Trust guidelines are being followed, to some degree. $25 \%$ of ultra-sounded sacral dimples were not adequately described on referral. A significant number of SU are still done for low risk dimples and these can be safely avoided reducing cost and parental anxiety.

\section{G312(P) SINGLE CENTRE, MULTI-LOCATION, INTERPROFESSIONAL REAL TIME OUTREACH SIMULATION}

${ }^{1} \mathrm{CE}$ Wensley, ${ }^{2} \mathrm{~T}$ Stephenson, ${ }^{1} \mathrm{GC}$ Millman. ${ }^{1}$ Paediatric, York Teaching Hospitals Trust, York, UK; ${ }^{2}$ Paediatric, Hull and East Yorkshire Hospitals NHS Trust, Hull, UK 